\title{
Introducing uncertainty of complex- valued quantities in measurement science education
}

Dailys Arronde Pérez, Hubert Zangl

Dailys Arronde Pérez, Hubert Zangl, "Introducing uncertainty of complexvalued quantities in measurement science education," Proc. SPIE 11144, Photonics and Education in Measurement Science 2019, 1114410 (17 September 2019); doi: 10.1117/12.2531840

EDIE Event: Joint TC1 - TC2 International Symposium on Photonics and Education in Measurement Science 2019, 2019, Jena, Germany 


\title{
Introducing Uncertainty of Complex-Valued Quantities in Measurement Science Education
}

\author{
Dailys Arronde Pérez ${ }^{\mathrm{a}}$ and Hubert Zangla \\ ${ }^{a}$ Institute of Smart Systems Technologies, Sensors and Actuators, Alpen-Adria-Universität \\ Klagenfurt, Universitätstrasse 65-67, 9020 Klagenfurt, Austria
}

\begin{abstract}
Uncertainty evaluation plays a key role in assessing and comparing measurement results, e.g. towards the design of experiments and the design of measurement systems. Nevertheless, it is frequently neglected by engineers particularly in early design phases, which may cause problems during the design process or even lead to system failures. In prior work, the use of software tools for uncertainty calculation in measurement science education has been suggested in order to raise awareness and increase the use of such tools to obtain GUM conform uncertainty estimates. However, we found that uncertainty of complex-valued quantities represents a major challenge in this context. Therefore, we present a proposal to extend the teaching concept towards such quantities, based directly on the utilization of an "Uncertainty Toolbox" for MATLAB maintained by our research group. The approach evaluates the uncertainty in complex parameters considering the real and imaginary components separately, with potential correlations between them arising from shared input quantities. As teaching example we study the Maxwell-Wien Bridge, as it is commonly taught in measurement science courses and brings in aspects such as above mention correlations and different representations of the measurement result (magnitude/phase, real/complex part of impedance). Based on this example, advantages and disadvantages of the presented teaching philosophy are discussed, emphasizing how problems arising from uncertainty may be identified in early design phases also considering complex-valued quantities.
\end{abstract}

Keywords: Uncertainty, complex-valued quantities, measurement, software toolbox, AC Bridge.

\section{INTRODUCTION}

Measurement science is an important part of modern engineering, being essential in almost all spheres of electronic industry. With respect to metrology, the quality of measurement results has been expressed in terms of uncertainty. This uncertainty quantifies the lack of knowledge or doubt about the validity of the result of a measurement, that is usually composed by realization of random variables. In order to provide an international consensus for evaluating and expressing uncertainties, the Guide to the Expression of Uncertainty in Measurement (GUM) 1 was developed. One of the initial requirements for this recommendation is that the approach has to be universal, so the GUM treats all uncertainty contributions identically, more or less as if the distributions were Gaussian and the relations were linear. According to the GUM, uncertainty is the parameter associated with the result of a measurement, that characterizes the dispersion of the values that could reasonably be attributed to the measurand. However, the GUM has some limitations. For instance, it does not contemplate the case of measurements involving complex-valued quantities, which was included in supplement 2 (2). A method for propagating uncertainty in complex parameters has also been addressed in Refs. 3 and 4, where the multivariate form of the error propagation law is explored. This approach deals with the real and imaginary components as distinct measurands and the correlation between them, arising from shared influence input quantities.

In some applications, when the quantity of interest as well as the parameters on which it depends are complex to define the measurement model in term of its real and imaginary functions can be a tough task. Fortunately, tools for uncertainty calculation are available (5). Also "An Uncertainty Toolbox" for Matlab has

Further author information:

D.A.P.: E-mail: dailys.arronde@aau.at, Telephone: +43 46327003553

H.Z.: E-mail: hubert.zangl@aau.at, Telephone: +43 46327003562

Photonics and Education in Measurement Science 2019, edited by Maik Rosenberger,

Paul-Gerald Dittrich, Bernhard Zagar, Proc. of SPIE Vol. 11144, 1114410

(C) 2019 SPIE · CCC code: 0277-786X/19/\$21 · doi: 10.1117/12.2531840 
been developed by our research group. This software toolbox includes the implementation of the GUM tree method with automatic differentiation. Its basic concept and additional aspects such as numeric representation of uncertainty have been discussed in Ref. 6 .

Teaching philosophies addressing uncertainty in scalar-valued quantities and robust design principles for reliability and safety measurement system, have been proposed in Ref. 7 and 8 respectively. In electrical measurements, many parameters of interest are complex-valued. That's why we introduce an example of the Maxwell-Wien Bridge to our students. This simple example is suitable for discussing pros and cons of using such uncertainty toolbox with complex quantities and also for enhancing the awareness with respect to uncertainty and how it can impact the overall outcome in measurement chains.

\section{TEACHING CONCEPT}

The course in question, Measurement Science, Sensors and Actuators, target students in the third year of Information Technology. Accordingly, it is expected that they are familiar with concepts of electrical measurements, complex variables calculus, probability theory and stochastic. The course outline is illustrated in Table 1 . We decide to start the course introducing the concept of uncertainty, interpreting measurements as realization of random variables provides some information about the parameter in question. We also introduce the original GUM including terms such as combined standard uncertainty and its determination by means of Taylor series expansion. The extension of this term for complex measurements is studied through the "Uncertainty Toolbox" for Matlab developed and maintained in our research group. It implements the GUM tree method, considering an extension of the error propagation law for complex parameters.

Table 1: Outline for introducing the concept of uncertainty with complex quantities.

\begin{tabular}{|l|l|}
\hline \multicolumn{1}{|c|}{ Section } & Content \\
\hline \hline 1.Basic Concepts & $\begin{array}{l}\text { Introduction to Uncertainty, GUM and its application to measure- } \\
\text { ment equation. Presentation of uncertainty toolbox in Matlab }\end{array}$ \\
\hline \hline $\begin{array}{l}\text { 2.Defining complex un- } \\
\text { certain quantities }\end{array}$ & $\begin{array}{l}\text { Limitation of the original GUM regarding complex measurements. } \\
\text { Uncertainty for complex measurements, supplement 2 of GUM. } \\
\text { Defining complex quantities with the uncertainty toolbox. }\end{array}$ \\
\hline \hline $\begin{array}{l}\text { 3.Example Maxwell- } \\
\text { Wien Bridge }\end{array}$ & $\begin{array}{l}\text { Pitfalls in the classic balance equation. Finding a suitable mea- } \\
\text { surement model. Implementation using the uncertainty toolbox } \\
\text { with Matlab }\end{array}$ \\
\hline \hline 4.Discussion & $\begin{array}{l}\text { Advantages of using the Toolbox. Generalization of the concept } \\
\text { of uncertainty for complex measurements. }\end{array}$ \\
\hline
\end{tabular}

\section{COMPLEX-VALUED QUANTITIES USING THE UNCERTAINTY TOOLBOX}

Figure 1 exemplifies how to define a complex quantity as uncertain object. First, the real and imaginary components are declared independently, e.i the real part $x$ has value $=3$, standard uncertainty $=0.2$ and name 'real_Z'. Then, the function complex is used to create the uncertain complex quantity $Z$.

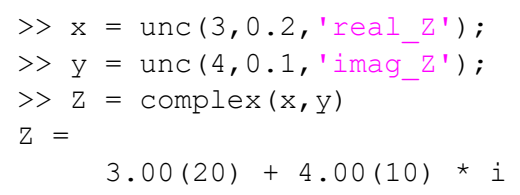

Figure 1: Introductory example, defining complex-valued quantities with the uncertainty toolbox in Matlab. Note that the real and imaginary components with their respective standard uncertainties should be defined independently. 
Complex numbers can be expressed in Cartesian and/or Polar representation. It is noteworthy to highlight how the standard uncertainty can be interpreted in both cases. Figure 2 shows the uncertain complex quantity $\mathrm{Z}$ in the complex plane. In the Cartesian case, we can easily represent the values and uncertainties of the real and imaginary parts $x$ and $y$. We can also interpret them as realizations of random variables, with mean and standard deviation directly associated with the properties value and standard uncertainty respectively. In the Polar case, it is not so intuitive to make such analogy, but the uncertainty can be geometrically represented as shows Figure 2.

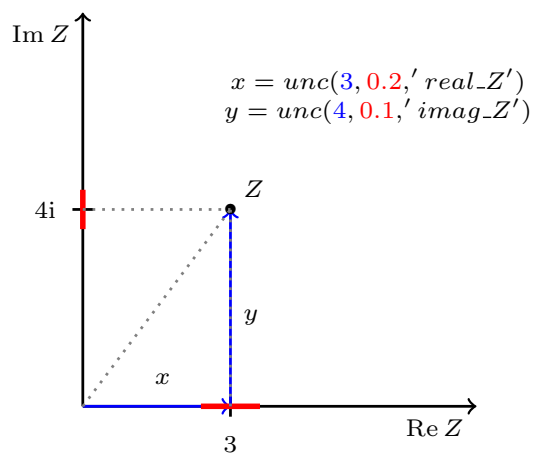

(a) Cartesian form

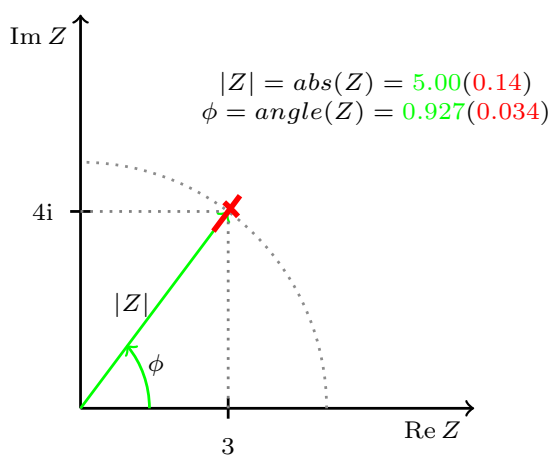

(b) Polar Form

Figure 2: Geometric representation of the uncertain complex quantity $\mathrm{Z}$ in the complex plane.(a) Cartesian representation of $Z$. Values of the real and imaginary parts are depicted in blue and the corresponding uncertainties, in red. (b) Polar representation of $\mathrm{Z}$. The magnitude and phase of $\mathrm{Z}$ are computed with the toolbox using the functions abs and angle, respectively. The values are depicted in green and the standard uncertainty, in red.

In the next section we introduce an example of the Maxwell-Wien bridge, as an extension of the Wheatstone Bridge circuit addressed in Ref.7 towards the complex domain.

\section{THE MAXWELL-WIEN BRIDGE WITH UNCERTAINTIES}

Bridge circuits are well-known topologies usually introduced in courses of measurement science, due to its wide application for the determination of unknown impedances and as realization of compensation method. AC bridges can be used to measure the value of inductance, capacitance and/or frequency. The Maxwell-Wien bridge is found to be more suitable for measuring unknown inductance (usually with low quality factor) and its circuit is shown in Figure 3. We introduce the Maxwell-Wien Bridge to emphasize how this toolbox for uncertainty calculation may change the way to present this subject to students and also as a suitable example for showing how to use the toolbox involving complex quantities.

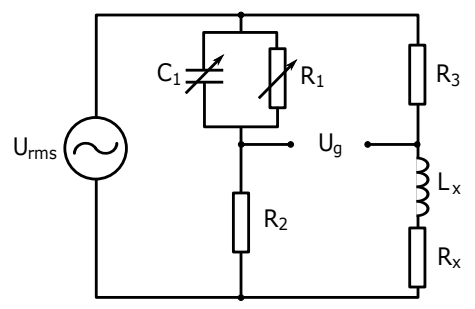

Figure 3: Example for a Maxwell-Wien Bridge.

If we consider the general $\mathrm{AC}$ bridge balance equation, the value of the unknown impedance $Z_{x}$ can be determinated as

$$
Z_{x}=Z_{2} \frac{Z_{3}}{Z_{1}}
$$


where $Z_{2}=R_{2}, Z_{3}=R_{3}, Z_{x}$ and $Z_{1}$ are complex quantities given by $Z_{x}=R_{x}+j w L_{x}$ and $\frac{1}{Z_{1}}=\frac{1}{R_{1}}+j w C_{1}$ respectively, with $w$ representing the angular frequency of the power supply with effective voltage $U_{r m s}$. To determinate the corresponding combined standard uncertainty of $Z_{x}$ we could think in directly applying the GUM method for uncorrelated inputs to the measurement model in (1), leading to

$$
u_{c}^{2}\left(Z_{x}\right)=\frac{\partial Z_{x}^{2}}{\partial Z_{2}} u^{2}\left(Z_{2}\right)+\frac{\partial Z_{x}^{2}}{\partial Z_{3}} u^{2}\left(Z_{3}\right)+\frac{\partial Z_{x}^{2}}{\partial Z_{1}} u^{2}\left(Z_{1}\right)
$$

and let the students do an interpretation of the results. The aim is to highlight two important issues. The first one is related to the fact of propagating uncertainty of complex-valued quantities, where the bivariate form of the Gaussian error propagation law 3 , should be considered. Then $Z_{x}$ is expressed in terms of its real and imaginary components as

$$
\mathbf{Z}_{x}=\left[\begin{array}{c}
R_{x} \\
w L_{x}
\end{array}\right]=\left[\begin{array}{c}
\frac{R_{2} R_{3}}{R_{1}} \\
w R_{2} R_{3} C_{1}
\end{array}\right]
$$

and its covariance matrix is given by

$$
\mathbf{C}\left(\mathbf{Z}_{x}\right)=\left[\begin{array}{cc}
u^{2}\left(R_{x}\right) & u\left(R_{x}, w L_{x}\right) \\
u\left(R_{x}, w L_{x}\right) & \omega^{2} u^{2}\left(L_{x}\right)
\end{array}\right]
$$

obtained by applying the error propagation law in the general matrix form, addressed in 9. The uncertainties of the real and imaginary parts of $Z_{x}, u^{2}\left(R_{x}\right)$ and $u^{2}\left(w L_{x}\right)$ respectively, could be found by applying the GUM method for scalar quantities. It is valid to clarify that the standard uncertainty of $Z_{x}$ it is not a complex quantity and can be expressed as

$$
u_{c}^{2}\left(Z_{x}\right)=u^{2}\left(R_{x}\right)+\omega^{2} u^{2}\left(L_{x}\right)
$$

It proceeds from the fact that in multivariate statistics the total variance is described as the trace of the covariance matrix, i.e. it ignores correlations.

The second issue to point out is that apparently some important influences are missing. From the measurement model in (1), it could be induced that the choice of the AC voltage source and the accuracy of the instrument that measures $U_{g}$ will have no impact on the resulting uncertainty. This is obviously not correct, which leads to do a more detailed analysis in order to find a suitable measurement model that considers the influences of $U_{r m s}$ and $U_{g}$. In general, to fully determine the standard uncertainty it may not be sufficient to only consider a measurement equation.

Consequently, the students are instructed to derive a more complete measurement model such as

$$
Z_{x}=\frac{U_{r m s} Z_{3}\left(Z_{1}+Z_{2}\right)}{U_{r m s} Z_{1}+U_{g}\left(Z_{1}+Z_{2}\right)}-Z_{3}
$$

Here we emphasize that despite the value of $U_{g}$ is measured to be zero, it may still be significantly different from zero and must not be omitted for uncertainty considerations. In order to find an expression for the combined standard uncertainty of $Z_{x}$, we first should express (6) in terms of the known parameters, i.e.

$$
Z_{x}=\frac{U_{r m s} R_{3}\left(R_{1}+R_{2}+j w R_{1} R_{2} C_{1}\right)}{U_{r m s} R_{1}+U_{g}\left(R_{1}+R_{2}+j w R_{1} R_{2} C_{1}\right)}-R_{3} .
$$

Then, the expressions for $R_{x}=\operatorname{real}\left(Z_{x}\right)$ and $\omega L_{x}=\operatorname{imag}\left(Z_{x}\right)$ are derived with respect to $U_{r m s}, U_{g}, C_{1}, R_{1}, R_{2}, R_{3}$, leading to the corresponding sensitivity coefficients $C_{r_{R_{1}}}=\frac{\partial R_{x}}{\partial R_{1}}, C_{i_{R_{1}}}=\frac{\partial L_{x}}{\partial R_{1}}, C_{r_{R_{2}}}=\frac{\partial R_{x}}{\partial R_{2}}$ and so on. Therefore, the resulting standard uncertainty of $R_{x}$ and $\omega L_{x}$ are given by

$$
\begin{aligned}
& u^{2}\left(R_{x}\right)=C_{r_{U_{r m s}}}^{2} u^{2}\left(U_{r m s}\right)+C_{r_{U_{g}}}^{2} u^{2}\left(U_{g}\right)+C_{r_{C_{1}}}^{2} u^{2}\left(C_{1}\right)+C_{r_{R_{1}}}^{2} u^{2}\left(R_{1}\right)+C_{r_{R_{2}}}^{2} u^{2}\left(R_{2}\right)+C_{r_{R_{3}}}^{2} u^{2}\left(R_{3}\right) \\
& \omega^{2} u^{2}\left(L_{x}\right)=C_{i_{U_{r m s}}}^{2} u^{2}\left(U_{r m s}\right)+C_{i_{U_{g}}}^{2} u^{2}\left(U_{g}\right)+C_{i_{C_{1}}}^{2} u^{2}\left(C_{1}\right)+C_{i_{R_{1}}}^{2} u^{2}\left(R_{1}\right)+C_{i_{R_{2}}}^{2} u^{2}\left(R_{2}\right)+C_{i_{R_{3}}}^{2} u^{2}\left(R_{3}\right)
\end{aligned}
$$


where the uncertainty $u(X)$ of the respective input quantities were previously calculated. Considering the result in (5), we can get the uncertainty associated to the complex quantity $Z_{x}$. However, the procedure for splitting equation (7) in its real and imaginary components can be difficult to perform manually and it is very likely that calculation errors are made, as well as in the derivation of the sensitivity coefficients.

Hence the next step is to introduce the uncertainty toolbox for Matlab to obtain the value and uncertainty of the unknown parameter $Z_{x}$. Figure 4 shows how to implement the procedure explained above step by step. Note that the equivalent impedance $Z_{1}$ is declared in terms of its real an imaginary parts, as was explained previously. Nevertheless, to obtain the combined standard uncertainty of the unknown impedance it does not require any additional effort, but providing the standard uncertainty for the input quantities. The last command line returns the numeric value of $u_{c}\left(Z_{x}\right)$.

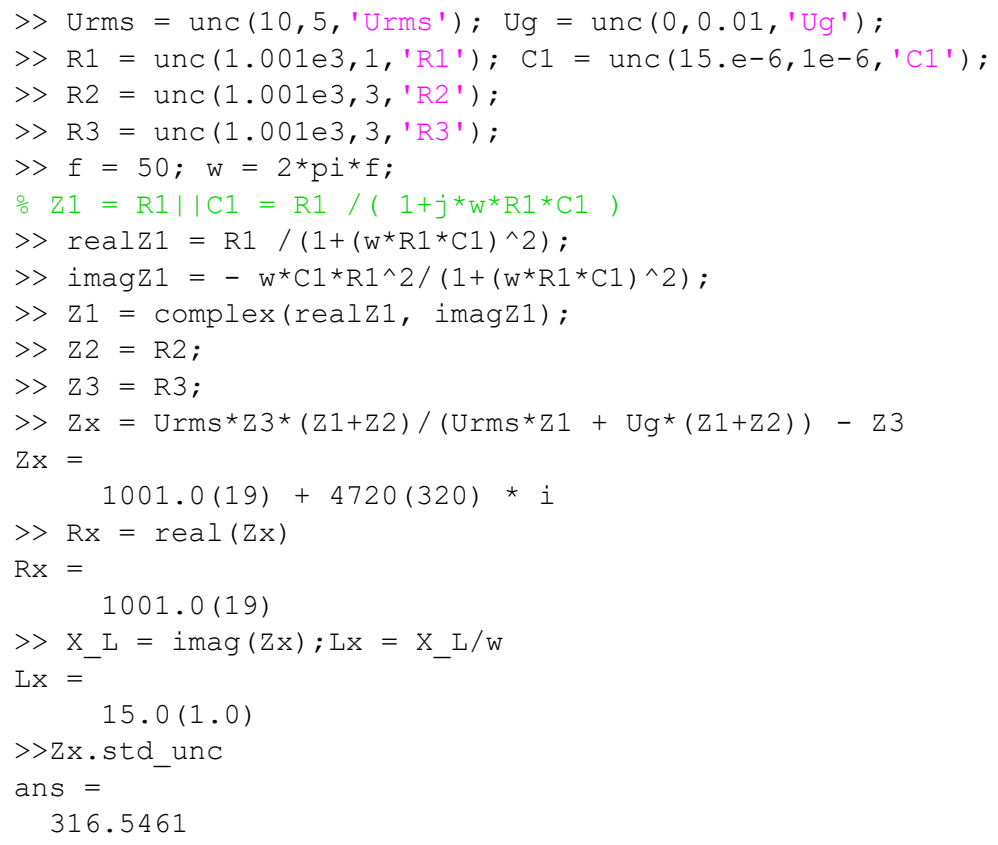

Figure 4: Matlab code to calculate the unknown impedance $Z_{x}$ and the standard uncertainty using the uncertainty toolbox.

In Figure 5 is presented the contributions of the input variables to the standard uncertainties of $R_{x}$ and $L_{x}$, showing that the main contributions come from the uncertainties of the measured voltage $\left(U_{g}\right)$ and the capacitor $\left(C_{1}\right)$ respectively. The reported values represent the square root of the contribution of each input quantity to the squared standard uncertainty of the output.

Figure 6 depicts how to obtain the covariance and correlation matrix associated with $R_{x}$ and $L_{x}$. The correlation coefficient between the real and imaginary components of the impedance $Z_{x}$ is not 0 due to the shared input parameters. With this example can be highlighted that the benefit of using the toolbox is not merely the automatic calculation of the unknown values and its associated uncertainties, it also provides means to analysis how the different sources of uncertainty contribute to the final result.

For comparative purposes, we also use the toolbox applying the model in (1).(See Figure 7). We obtain the same result for the unknown impedance, with a change in the standard uncertainty. This is because the main contribution to the uncertainty becomes from $U_{g}$, despite the fact that the contribution of $U_{r m s}$ can be neglected, as was shown in Figure 5 .

With the help of the toolbox we can easily study the dependencies between variables and how a change in one of the input parameters can influence the contribution of other inputs, as well as the combined standard uncertainty of the result. 

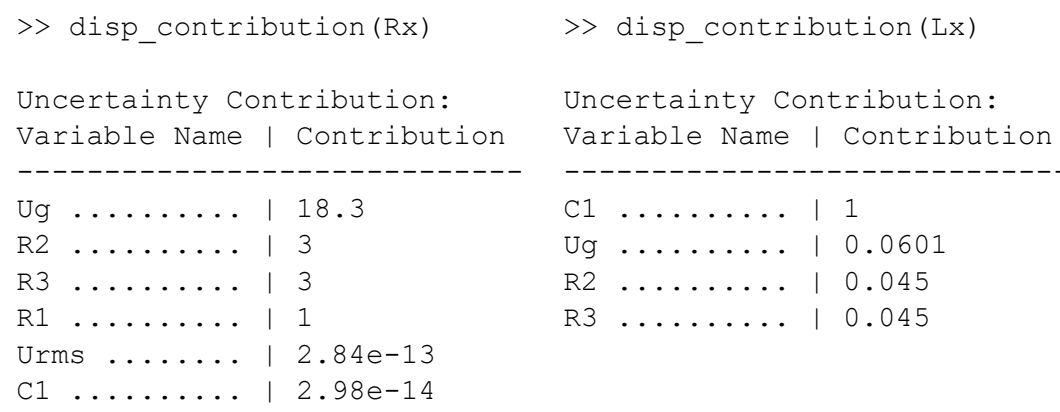

Figure 5: Determination of the contributions of input variables to the standard uncertainty of $R_{x}$ and $L_{x}$ using Matlab and the uncertainty toolbox.

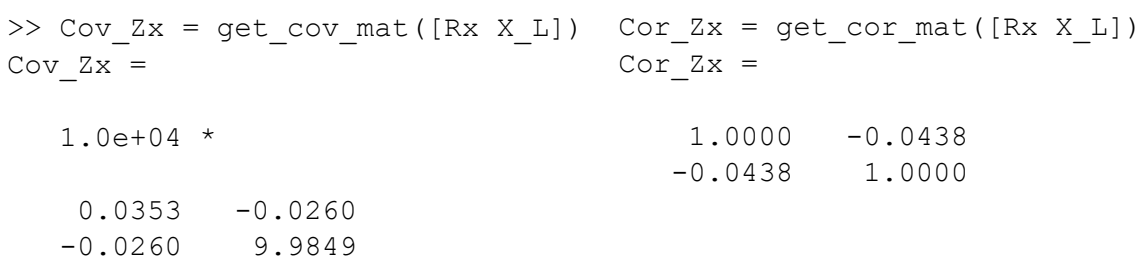

Figure 6: Determination of the Covariance and Correlation matrices of the vector $\mathbf{Z}_{x}$ using Matlab and the uncertainty toolbox.

\section{DISCUSSION OF ADVANTAGES AND DISADVANTAGES}

Measurement results can be shown as realizations of random variables and the GUM tries to describe the probability density function using the standard uncertainty. Therefore, our aim is to put the focus on the fact that uncertainty in the measurement of complex-valued quantities can be analyzed in terms of its real and imaginary components, as a realization of a 2-dimensional random vector. We consider a software toolbox for the automatic calculation of the combined standard uncertainty involving complex measurements. One of the main advantages of using the toolbox is that the students' attention can be guided towards the measurement model construction and the study of the sources of uncertainty rather than in the calculations itself. Another important point is that students can easily improve their abilities using the toolbox, since Matlab environment is acquainted for them.

An observed disadvantage is that sometimes students can experience a lack in the notion whether a result is in the correct order of magnitude, since the toolbox doesn't take into account the SI, as with electronic calculators in general. On the other hand, the toolbox uses the GUM approach 2, based on a first-order Taylor series approximation (only the complex derivatives of first order are needed) i.e. all uncertainty contributions are treated as if their relations were linear. Therefore for measurement models with significant non-linearity, the results given by the toolbox may be inaccurate. Such limitations are no intuitive for students and must be emphasized.

\section{CONCLUSION}

A teaching concept for considering uncertainty in complex-valued quantities have been proposed. The approach is based on the utilization of an "Uncertainty Toolbox" in Matlab which implements the GUM method, involving either scalar or complex-valued quantities and supporting different measurement models. Using this approach in measurement science education may help to increase the awareness with respect to uncertainty, leading to its consideration in early design phases which can contribute to the robustness and reliability of systems. 
